\title{
Abundância do Guariba-de-mãos-ruivas (Alouatta belzebul) na Reserva Biológica Guaribas, Paraíba, Brasil
}

\author{
Anderson Coimbra ${ }^{1}$, Gerson Buss ${ }^{2} \&$ Renata Bocorny de Azevedo ${ }^{2}$
}

Recebido em 25/11/2020 - Aceito em 10/05/2021

${ }_{1}^{1}$ Universidade Federal da Paraíba/UFPB, Brasil. <anderson15ou@gmail.com>
${ }^{2}$ Instituto Chico Mendes de Conservação da Biodiversidade/CPB/ICMBio, Brasil. <gbuss.icmbio@gmail.com, renata.azevedo@icmbio.gov.br >

RESUMO - O guariba-de-mãos-ruivas (Alouatta belzebul) é um primata com ocorrência restrita à porção oriental da Floresta Amazônica e à Mata Atlântica do nordeste brasileiro. Encontra-se ameaçado de extinção, principalmente devido à situação das populações nordestinas da espécie. Em 1990, foi criada a Reserva Biológica Guaribas (REBIO Guaribas), com 4.028,25ha, para proteger o habitat desta espécie que, na época, estava localmente extinta. Em 1998 foi iniciado o Projeto "Guaribas do Nordeste" com o objetivo de repovoar a área através da translocação de animais. Considerando que os números atuais da população de guaribas não são conhecidos, esse estudo teve por objetivo determinar a abundância e densidade populacional do guariba-de-mãos-ruivas (Alouatta belzebul) na REBIO, através do método de transecção linear. Foram utilizadas duas trilhas, totalizando $7 \mathrm{~km}$ de extensão. A cada mês, as trilhas foram percorridas três vezes cada, no período da manhã, entre 06:30 e 10:30, a uma velocidade média de 1,7km/h. Em dez meses foram percorridos $210 \mathrm{~km}$, sendo obtida uma taxa de encontro de 0,38 vis. $/ 10 \mathrm{~km}$. Devido ao pequeno número de visualizações, não foi possível estimar a densidade. Entretanto, os resultados deste estudo indicam que a abundância de A. belzebul na REBIO Guaribas é baixa, quando comparada com outros estudos. Entende-se que as ações de reintrodução do $A$. belzebul na REBIO Guaribas têm sido bem-sucedidas no estabelecimento de uma população, que apesar de pequena, está conseguindo se manter e reproduzir. Sugere-se a continuidade das ações de repovoamento, a fim de garantir a conservação desse primata ameaçado nessa importante área da Mata Atlântica nordestina.

Palavras-chave: Primatas; espécie ameaçada; Mata Atlântica; reintrodução; nordeste.

\section{Abundance of the Red-handed Howler (Alouatta belzebul) in the Guaribas Biological Reserve, Paraíba, Brazil}

\begin{abstract}
The red-handed howler (Alouatta belzebul) is a primate with occurrence restricted to the eastern portion of the Amazon Forest and the northeastern Atlantic Forest of Brazil. It is threatened with extinction mainly due to the situation of the northeastern populations of the species. In 1990, the Guaribas Biological Reserve (REBIO Guaribas) was created, with 4,028.25 ha, to protect the habitat of this species that was locally extinct. In 1998 the "Guaribas do Nordeste" Project (Northeastern Howlers Project) started with the objective of reintroducing the howlers in the area through animal translocation. Considering that the current population size of howlers is unknown, this project aimed to determine both the abundance and the population density of howlers in the REBIO using the linear transect method. Two trails were used, totaling $7 \mathrm{~km}$ in length. The trails were walked three times each month, during the morning, between 6:30 am and 10:30 am, at an average speed of $1.7 \mathrm{~km} / \mathrm{h}$. A total of $210 \mathrm{~km}$ were walked through ten months, and an encounter rate of 0.38 sightings $/ 10 \mathrm{~km}$ was obtained. Due to the small number of encounters, it was not possible to estimate the density. However, the results from this study indicate that the abundance of $A$. belzebul in the REBIO Guaribas is low compared with other studies. Besides, the reintroduction efforts have successfully established a population that - although small - is being able to persist and reproduce. Continuing population reinforcement is suggested to guarantee the conservation of this threatened primate in such an important northeastern Atlantic Forest remnant.
\end{abstract}

Keywords: Primates; endangered species; atlantic forest; reintroduction; northeast. 


\title{
Abundancia de Mono Aullador de Manos Rojas (Alouatta belzebul) en la Reserva Biológica Guaribas, Paraíba, Brasil
}

\begin{abstract}
RESUMEN - El mono aullador de manos rojas (Alouatta belzebul) es un primata con ocurrencia restringida a la porción oriental de la selva amazónica y mata atlántica del nordeste brasileño. Se encuentra amenazada de extinción, principalmente por la situación de las poblaciones del nordeste. En 1990 fue creado la Reserva Biológica Guaribas (REBIO Guaribas), com 4.028,25ha, para proteger el hábitat de esta especie que, en ese momento, estaba localmente extinta. En 1998 empezó lo proyecto "Guaribas do Nordeste" con el objetivo de repoblar la zona mediante la translocación de individuos mono aullador. Considerando que no se conocen la cantidad actuales de la población de mono, esto estudio tuvo como objetivo determinar la abundancia y densidad de población del mono aullador de manos rojas en la REBIO, a través del método de transección lineal. Dos caminos fueron utilizados con un total de $7 \mathrm{~km}$. Cada mes los caminos eran hechos tres veces cada por la mañana entre 6:30 y 10:30 a una velocidad media de $1,7 \mathrm{~km} / \mathrm{h}$. En diez meses $210 \mathrm{~km}$ fueron atravesados obteniendo una tasa de encuentro de $0,38 \mathrm{vis} . / 10 \mathrm{~km}$. Debido al pequeño número de vistas, no fue posible estimar la densidad. Estos resultados indican que la abundancia de A.belzebul en la REBIO Guaribas es baja cuando se compara con otros estudios. Se entiende que las acciones de reintrodución de A.belzebul tuvieron éxito en establecer una población que a pesar de ser pequeña está logrando éxito en mantenerse y reproducirse. Se sugiere continuar con las acciones de reintroducción, con el fin de garantizar la conservación de este primate en peligro de extinción en la mata atlántica del nordeste del Brasil.
\end{abstract}

Palabras-clave: Primates; especie en peligro; mata atlántica; reintrodución; nordeste.

\section{Introdução}

O guariba-de-mãos-ruivas (Alouatta belzebul) é um primata neotropical de grande porte que possui cauda preênsil, além do grande complexo hióde (Neville et al., 1988), responsável pela sua alta vocalização. $\mathrm{O}$ peso de um indivíduo pode variar de $3,2 \mathrm{~kg}$ (fêmea) a 4,7kg (macho) (Valença-Montenegro et al., 2018) e seu hábito alimentar folívoro-frugívoro os faz pouco ativos, repousando cerca de $66 \%$ do dia (Camargo et al., 2008). Além disso, como outros primatas do gênero Alouatta, desempenha um papel de dispersor primário de sementes que beneficia a distribuição das espécies arbóreas e a regeneração florestal (Bonvicino, 1989; Julliot, 1996).

A espécie é endêmica ao Brasil, sendo restrita à porção oriental da Floresta Amazônica e na Mata Atlântica do nordeste brasileiro (Gregorin, 2006), apresentando distribuição disjunta (ValençaMontenegro et al., 2018). A Floresta Amazônica e a Mata Atlântica estão entre os ecossistemas com os maiores índices de biodiversidade do mundo, $e$ estão separadas pela diagonal de áreas abertas da América do Sul, conhecida também como Diagonal Seca, que é formada pelos biomas Chaco, Cerrado e Caatinga. Acredita-se que a Floresta Amazônica e a Mata Atlântica foram conectadas várias vezes através desta diagonal seca durante períodos climáticos mais úmidos que o atual nesta região, como o final do Pleistoceno. São consideradas evidências do processo de retração e expansão, a presença de enclaves de florestas úmidas no interior da Caatinga (Zanella, 2011). Baseado em padrões de distribuição de mamíferos, no registro fóssil e na ecofisiologia, De Vivo e Carmignotto (2004) sugerem que uma floresta de brejo capaz de suportar mamíferos arbóreos de florestas tropicais existiu na área presentemente ocupada pela Caatinga, ligando Amazônia Oriental $e$ as florestas tropicais do Atlântico.

Nonordeste brasileiro, A. belzebulestá presente em 18 fragmentos de Mata Atlântica espalhados por quatro estados: Alagoas, Pernambuco, Paraíba e Rio Grande do Norte (Fialho et al., 2014). Atualmente, a Mata Atlântica nordestina apresenta apenas $12 \%$ de sua cobertura original, representando um grande impacto para a conservação de animais na região, principalmente primatas. Das sete espécies que habitam a região, quatro estão ameaçadas de extinção, incluindo o guariba-de-mãos-ruivas categorizado como Vulnerável (MMA, 2014; Valença-Montenegro et al., 2018).

Considerando que a maioria das populações de A. belzebul encontra-se em áreas desprotegidas, em 1990 foi criada a Reserva Biológica Guaribas (REBIO Guaribas), localizada no município de Mamanguape/PB, com o objetivo de proteger o hábitat desta espécie que, na época, estava 
localmente extinta (Coimbra-Filho et al., 1995). Assim, a partir de 1998, foi iniciado o Projeto "Guaribas do Nordeste" com o objetivo de reintroduzir a espécie na REBIO Guaribas, através da translocação de indivíduos que viviam em áreas ameaçadas, principalmente oriundos da Fazenda Pacatuba, e da soltura de guaribas apreendidos pelo IBAMA. Entre 1998 a 2008, 12 guaribas foram liberados na REBIO Guaribas começando assim, a reintrodução (Damião, 2011). Através do método de busca ativa, Damião (2011) estimou a ocorrência de pelo menos 21 indivíduos na REBIO Guaribas, distribuídos em cinco grupos. Após essa estimativa, no período de outubro de 2011 a julho de 2017 foram liberados mais 12 indivíduos em diferentes ações de manejo do Centro Nacional de Pesquisa e Conservação de Primatas Brasileiros (ICMBio/CPB) (Gabriela Ludwig, comunicação pessoal, 29 de setembro de 2020).

A REBIO faz parte de uma das áreas importantes para a conservação dos primatas do Nordeste, definidas como espaços geográficos que contemplam registros de ocorrência de A. belzebul e/ou Sapajus flavius onde qualquer intervenção de origem antrópica trará, potencialmente, implicações diretas para a conservação da população selvagem remanescente mais próxima $e$, em última instância, a conservação dessas espécies como um todo" (CPB, 2014). Para as populações de $A$. belzebul foram definidas dez áreas importantes.

Além disso, o guariba-de-mãos-ruivas é uma das seis espécies contempladas no Plano de Ação Nacional para a Conservação dos Primatas do Nordeste (PAN PRINE), uma política pública que orienta as ações prioritárias para minimizar as ameaças a essas espécies. O PAN PRINE foi elaborado em 2018 e tem como objetivo manter e promover a viabilidade de populações das espécies alvo em cinco anos (ICMBio, 2020).

Considerando que os números atuais da população de guaribas existente na REBIO não são conhecidos, este estudo teve por objetivo determinar a abundância e densidade populacional do guariba-de-mãos-ruivas (A. belzebul) estabelecendo, dessa forma, um marco inicial no monitoramento desta população através do método de transecção linear e, contribuindo também para a avaliação da efetividade do repovoamento na REBIO Guaribas.

\section{Material e Métodos}

O estudo foi realizado na REBIO Guaribas (6 43' 17' S, 35 10' 49" O), localizada na zona rural da cidade de Mamanguape-PB e que protege um dos últimos remanescentes de Mata Atlântica no Estado da Paraíba (Figura 1). A REBIO foi criada em 1990, é administrada pelo Instituto Chico Mendes de Conservação da Biodiversidade (ICMBio) e divide-se em três áreas (SEMA 1, SEMA 2 e SEMA 3) inseridas em uma matriz dominada pela monocultura canavieira, com um total de 4.028,55 hectares (IBAMA, 2003). Esse estudo foi realizado na área denominada SEMA 2, com 3.016,09 hectares. A REBIO Guaribas se caracteriza por ter uma vegetação de floresta estacional semidecidual nos vales, intercalada por áreas de tabuleiro $e$ vegetação de savana arbórea aberta nas partes mais altas do relevo (Barbosa et al., 2011).

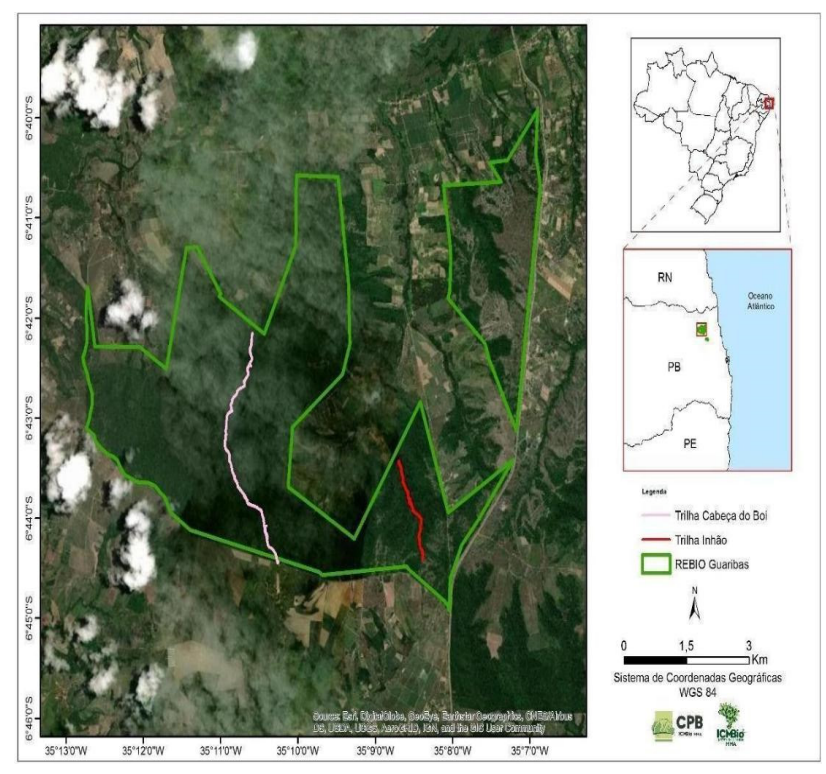

Figura 1 - Localização da REBIO Guaribas e das transecções utilizadas para este estudo.

A abundância da população de guaribade-mãos-ruivas na área foi acessada a partir do método de transecção linear (Burnham et al., 1980; Buckland et al.,1993; Peres \& Cunha, 2011). Foram utilizadas duas trilhas já existentes: trilha do Inhão $(2 \mathrm{~km})$ e trilha da Cabeça do Boi $(5 \mathrm{~km})$, totalizando $7 \mathrm{~km}$ de extensão. A cada mês as duas trilhas eram percorridas três vezes cada, no período da manhã, entre 06:30 e 10:30, a uma velocidade média de $1,7 \mathrm{~km} / \mathrm{h}$. Para cada visualização foi anotada a hora de encontro, 
nome da trilha, distância perpendicular entre o observador e o primeiro animal, ponto na trilha, altura estimada do primeiro animal, a quantidade de indivíduos e o sexo (quando possível).

A trilha do Inhão tem seu início próximo a sede da REBIO (200m aprox.) e da BR-101 podendo-se, em alguns momentos, ouvir os automóveis transitando. É cercada por mata fechada com vegetação típica de Mata Atlântica com árvores ultrapassando os 15 metros de altura. Além disso, possui declives em seu curso. A trilha da Cabeça do Boi se inicia na PB-071, uma rodovia perpendicular à BR-101, e que passa em frente a sede da REBIO delimitando seu limite nesta área. Além de compartilhar as mesmas características vegetais que a trilha do Inhão, em sua porção final, apresenta uma região aberta de tabuleiro onde as árvores, em sua maioria, são de pequeno porte. Ambas as trilhas eram utilizadas pelos moradores de uma vila que existiu décadas antes da criação da Unidade de Conservação.

Para realizar a estimativa da densidade populacional é necessária uma quantidade amostral igual ou superior a 40 visualizações para um cálculo confiável (Buckland et al., 1993). Considerando o número expressivamente menor neste estudo e a fim de evitar resultados não condizentes com a realidade, optamos por não fazer o cálculo de densidade $e$ apresentar apenas o valor da abundância com base nas visualizações obtidas. A abundância foi obtida pelo cálculo da taxa de encontro (TE), dada pela razão entre o número de encontros de grupos por $10 \mathrm{~km}$ percorridos.

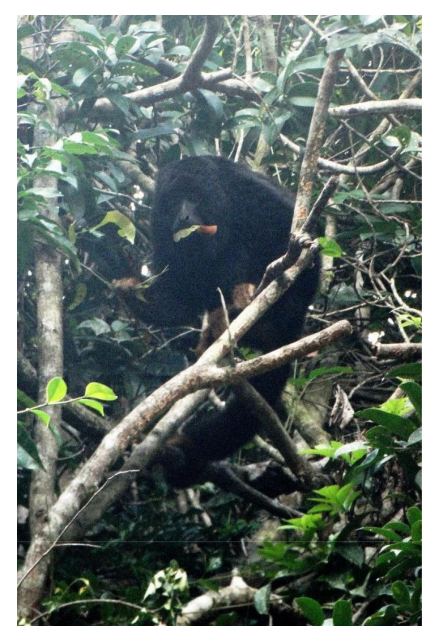

Figura 2 - Macho adulto de guariba-de-mãos-ruivas (Alouatta belzebul), espécie de primata ameaçado de extinção translocado para a REBIO Guaribas (PB) em julho de 2017. Foto: Gerson Buss.

\section{Resultados e Discussão}

Em dez meses de monitoramento (de setembro de 2017 a junho de 2018) foram realizadas 30 repetições em cada trilha resultando em $210 \mathrm{~km}$ percorridos. De acordo com Thoysi et al. (2008), esse esforço de campo é bastante adequado para uma estimativa robusta de abundância de primatas do gênero Alouatta. Nesse período foram registradas oito visualizações de guaribas-de-mãos-ruivas (A. belzebul) $e$ quatro do sagui-do-nordeste ( $C$. jacchus). Para $A$. belzebul foi obtida uma taxa de encontro de 0,38 vis. $/ 10 \mathrm{~km}$. Das oito visualizações registradas, sete foram na trilha do Inhão e apenas uma na trilha Cabeça do Boi.

Esses resultados indicam que a abundância de A. belzebul na REBIO Guaribas é baixa, quando comparada com os resultados de outros estudos na área de distribuição da espécie (Tabela 1). Na porção nordestina de sua distribuição, os valores de abundância de A. belzebul na REBIO Guaribas só não foram menores que os valores obtidos por Fialho \& Gonçalves (2008), na Reserva Particular do Patrimônio Natural Engenho Gargaú, localizada em Santa Rita/PB. Segundo Hue et al. (2016) a raridade de guaribas na RPPN Gargaú pode ser devido a caça, que até recentemente era comum na região. Considerando que já se passaram 13 anos deste estudo, seria interessante a realização de um novo estudo de abundância a fim de identificar a tendência populacional dos guaribas na área.

Tabela 1 - Comparação da abundância relativa do guariba-de-mãos-ruivas (Alouatta belzebul) em sua área de distribuição.

\begin{tabular}{|l|l|l|l|l|}
\hline $\begin{array}{c}\text { Área de } \\
\text { estudo }\end{array}$ & $\begin{array}{c}\text { Ab. rel. } \\
\text { (vis./10km) }\end{array}$ & $\begin{array}{c}\text { Esforço } \\
(\mathbf{K m})\end{array}$ & Área (ha) & Referência \\
\hline $\begin{array}{l}\text { REBIO } \\
\text { Guaribas, } \\
\begin{array}{l}\text { Mamanguape } \\
\text { (PB) }\end{array}\end{array}$ & 0,38 & 210 & 4.051 & Este estudo \\
\hline $\begin{array}{l}\text { Mata São João/ } \\
\text { Cafundó, } \\
\text { Santa Rita (PB) }\end{array}$ & 1,68 & 243 & 2.434 & $\begin{array}{l}\text { Santos Jr } \\
\text { \& Buss } \\
\text { (2016) }\end{array}$ \\
\hline $\begin{array}{l}\text { RPPN } \\
\text { Pacatuba, } \\
\text { Sapé (PB) }\end{array}$ & 12,7 & 48,1 & 266 & $\begin{array}{l}\text { Hue et al. } \\
\text { (2016) }\end{array}$ \\
\hline $\begin{array}{l}\text { RPPN Gargaú, } \\
\text { Santa Rita (PB) }\end{array}$ & 0,08 & 250 & 1.436 & $\begin{array}{l}\text { Fialho \& } \\
\text { Gonçalves } \\
\text { (2008) }\end{array}$ \\
\hline $\begin{array}{l}\text { REBIO do } \\
\text { Gurupi (MA) }\end{array}$ & 0,72 & 320 & 271.197 & $\begin{array}{l}\text { Buss et al. } \\
\text { (2017) }\end{array}$ \\
\hline
\end{tabular}




\begin{tabular}{l|l|l|l|l|}
$\begin{array}{l}\text { REBIO do } \\
\text { Gurupi (MA) }\end{array}$ & 0,56 & 480 & $271.197^{*}$ & $\begin{array}{l}\text { Lopes (2000) \& } \\
\text { Ferrari }\end{array}$ \\
\hline $\begin{array}{l}\text { Companhia } \\
\begin{array}{l}\text { Real Agro- } \\
\text {-Industrial, } \\
\text { Tailândia (PA) }\end{array}\end{array}$ & 0,05 & 216 & 17.485 & $\begin{array}{l}\text { Lopes \& Ferrari } \\
\text { (2000) }\end{array}$ \\
\hline
\end{tabular}

Na porção Amazônica de sua distribuição, o menor valor de abundância encontrado para A. belzebul $(0,05$ vis. $/ 10 \mathrm{~km})$ foi na área da Companhia Real Agro-Industrial, em Tailândia (PA), por Lopes \& Ferrari (2000), uma área com baixo distúrbio mas intensa pressão de caça. Já o maior valor $(0,56$ vis. $/ 10 \mathrm{~km})$ foi registrado na REBIO do Gurupi, com um baixo/moderado distúrbio e uma baixa/moderada pressão de caça (Lopes \& Ferrari, 2000). Em um estudo mais recente, na mesma localidade e também para $A$. belzebul, Buss et al. (2017) encontraram um valor de abundância de 0,72 vis. $/ 10 \mathrm{~km}$, mais alto que o registrado por Lopes \& Ferrari (2000). Segundo esses autores, esse resultado está relacionado aos esforços para implementação e proteção da Unidade de Conservação.

O número máximo de indivíduos visualizados, em um único encontro, foi de cinco guaribas, enquanto que o número médio foi de 3,25 indivíduos. Damião (2011) registrou um grupo com seis indivíduos na REBIO Guaribas. $\mathrm{Na}$ Mata São João-Cafundó, área com 2.434 ha, no município de Santa Rita (PB), Santos Jr. \& Buss (2016) encontraram um tamanho médio de grupo de cinco indivíduos. Na RPPN Pacatuba, Sapé (PB), com 256,33 ha a qual apresenta uma abundância extremamente alta (Hue et al., 2016), foram registrados grupos com uma média de 7,4 ind. (Bonvicino, 1989), além disso, Souza (2005) acompanhou um grupo com 16 indivíduos. A RPPN Pacatuba apresenta também uma abundância extremamente alta (Tabela 1) e, de acordo com Hue et al., (2016) isso pode ser explicado pela alta disponibilidade de árvores de grande porte e frutos nessa área.

Com relação à altura do estrato arbóreo, os guaribas foram registrados sempre acima de 10 metros. Esses resultados corroboram o observado por Hue et al., (2016) na RPPN Pacatuba.

Apesar dos esforços de conservação da equipe gestora da Unidade de Conservação, ainda são registradas algumas ameaças à integridade da REBIO. Queimadas afetam as bordas da Reserva, especialmente nas áreas de tabuleiro e de vegetação secundária (Barbosa et al., 2011). Além disso, são encontrados vestígios da presença de caçadores, principalmente na área conhecida como Cabeçado-boi. Porém, não tem sido registrada pressão de caça direcionada aos guaribas (Aluízio de Oliveira Silvestre, comunicação pessoal, Dezembro, 2017). Durante o estudo, foi registrada a presença de cães no interior da Unidade de Conservação, considerados predadores potenciais de primatas (Anderson, 1986, Galetti \& Sazima, 2006; Oliveira et al., 2008).

Levando-se em conta que sete das oito visualizações foram na trilha do Inhão, pressupõese que essa área concentre a maior parte da população de guaribas da REBIO. Garcia et al. (2014) em um estudo sobre o uso de playback para levantamento populacional de A.belzebul na REBIO Guaribas, obtiveram a maior frequência de respostas na região do "Inhão". Um fato que pode explicar essa alta incidência de visualizações é que o recinto pré-soltura fica localizado junto à sede da REBIO, próximo a esta trilha. Assim, além da continuidade das ações de reforço populacional, sugere-se que as próximas solturas sejam realizadas na região da "Cabeça do boi".

Considerando que a reintrodução de guaribas na REBIO é um processo relativamente recente (Damião, 2011), a baixa abundância era um resultado esperado. Bonvicino (1989) identificou que na RPPN Pacatuba (PB) o tamanho da área de vida de A. belzebul foi de 4,7 a 9,5 ha. Assim, considerando o número de guaribas translocados para a REBIO até o momento, o tamanho da área de vegetação arbórea presente, o tamanho da área de vida dos guaribas em área próxima (RPPN Pacatuba) e a baixa abundância encontrada, acredita-se que as ações de reforço populacional de A. belzebul na REBIO Guaribas devem continuar. Além disso, a maior abundância encontrada em fragmentos menores, como a Mata São João/Cafundó e a RPPN Pacatuba, pode indicar que a REBIO ainda suporta o aporte de mais indivíduos. A visualização de uma fêmea com filhote demonstra que uma população foi estabelecida, um importante indicativo de como as ações para o repovoamento estão sendo bemsucedidas.

Por fim, a utilização da transecção linear, uma metodologia amplamente conhecida, pode auxiliar o estabelecimento de um programa de monitoramento sistemático da população de guaribas na REBIO. Nesse sentido, a REBIO 
Guaribas deverá implementar, em breve, o protocolo básico do Componente Florestal do Programa Nacional de Monitoramento da Biodiversidade do ICMBio (Programa Monitora). Esse programa busca, a partir da cooperação entre pesquisadores, gestores e comunidades, através do monitoramento participativo nas Unidades de Conservação, levantar dados sobre a biodiversidade local para subsidiar projetos, avaliação da conservação da fauna e flora, sua ocorrência e como respondem às mudanças climáticas e outros fatores, alinhados ao baixo custo financeiro e operacional (ICMBIO 2021).

\section{Conclusão}

Considerando as visualizações obtidas e o registro de um filhote, entende-se que a reintrodução $e$ as ações de reforço populacional têm sido bemsucedidas, pois os guaribas têm conseguido se estabelecer e se manter na área da REBIO.

A implementação do Programa Monitora possibilitará que as populações de A. belzebul continuem sendo monitoradas periodicamente, permitindo avaliar a continuidade e efetividade das ações de manejo.

Os resultados desse estudo mostram que a abundância do guariba-de-mãos-ruivas (A. belzebul) ainda é baixa na REBIO Guaribas, sendo possível e desejável a continuidade das ações de reforço populacional, a fim de garantir a conservação desse primata ameaçado na Mata Atlântica nordestina.

\section{Agradecimentos}

Agradecemos ao Programa de Iniciação Científica do $\mathrm{CNPq} / \mathrm{ICMBio}$, à equipe da REBIO Guaribas, em especial a Getúlio Luís de Freitas, Severino Manuel Gomes (Seu Bil), Aluízio de Oliveira Silvestre, Damião Pedro da Silva e demais servidores, assim como aos brigadistas, pelo apoio no transporte e execução dos transectos.

Luciana G. Pacca, analista ambiental do $\mathrm{CPB}$, pela elaboração do mapa; ao Veterinário Plautino de Oliveira Laroque, pelo incentivo à execução deste trabalho $e$ aos dois revisores anônimos, pelas sugestões ao manuscrito.

\section{Referências}

Anderson J. Encounters between domestic dogs and freeranging non-human Primates. Applied Animal Behaviour Science, 15: 71- 86, 1986.

Barbosa, MRV, et al. Checklist of the vascular plants of the Guaribas Biological Reserve, Paraiba, Brazil. Rev. Nordestina Biol., 20(2): 79-106, 2011.

Bonvincino, CR. Ecologia e comportamento de Alouatta belzebul (Primates, Cebidae) na Mata Atlântica. Rev. Nordestina Biol., 9(2): 149- 179, 1989.

Buckland ST, Anderson DR, Burnham KP, Laake JL. 1993. Distance Sampling: estimating abundance of biological populations. Chapman and Hall, London, reprinted 1999 by RUWPA, University of St. Andrews, Scotland, 446p.

Burnham KP, Anderson DR, Laake JL. Estimation of density from line transect sampling of biological populations. Wildlife Monographs, 72: 1-201, 1980.

Buss $G$ et al. Abundância e densidade de primatas na Reserva Biológica do Gurupi, Maranhão, Brasil. Biodiversidade Brasileira, 7(2): 47-57, 2014.

Camargo, C.C., S. Porfírio, A.B. Rylands \& A. Langguth 2008. Variação sazonal e longitudinal nos padróes de comportamento em uma população de Alouatta belzebul (Primates: Atelidae) do Nordeste brasileiro pp192-201. In: A Primatologia no Brasil - 9 (S.F. Ferrari \& J. Rímoli, Eds.) Aracaju, Sociedade Brasileira de Primatologia, Biologia Geral e Experimental - UFS.

Coimbra-Filho AF, Câmara IG, Rylands $\mathrm{AB}$. On the Geographic Distribution of the Red-Handed Howling Monkey, Alouatta belzebul, in North-East Brazil. Neotropical Primates 3(4): 176-179, 1995.

CPB (Centro Nacional de Pesquisa e Conservação de Primatas Brasileiros), 2014. Áreas Importantes para a Conservação de Primatas do Centro de Endemismo Pernambuco. Instituto Chico Mendes para Conservação da Biodiversidade. João Pessoa. 47p.

Damião VLAG. 2011. Segundo relatório anual do Projeto Conservação do Guariba-de-Mãos-Ruivas da Mata Atlântica Nordestina: Ecologia e Demografia de uma População Reintroduzida e o seu Manejo de Reforço. CPB/ICMBio. 29p.

De Thoisy B, Brosse S, Dubois MA. Assessment of largevertebrate species richness and relative abundance in Neotropical forest using line-transect censuses: what is the minimal effort required? Biodivers Conserv., 17, 26272644, 2008.

De Vivo M \& Carmignotto AP. (2004). Holocene vegetation change and the mammal faunas of South America and Africa. Journal of Biogeography, 31(6), 943-957. 
Fialho MS, Gonçalves GF. Primatas da RPPN Gargaú, Paraiba, Brasil. Neotropical Primates, 15(2): 50-54, 2008.

Fialho MS, Valença-Montenegro MM, Silva TCF, Ferreira JC, Laroque PL. Ocorrência de Sapajus flavius $e$ Alouatta belzebul no Centro de Endemismo Pernambuco. Neotropical Primates 21(2): 214-219, 2014.

Galletti M, Sazima I. Impact of feral dogs in an urban Atlantic forest fragment in southeastern Brazil. Natureza \& Conservação, 4(1): 146-151, 2006.

Garcia, VLA, Fialho, MS, Jerusalinsky, L. 2014. Uso de playback para levantamento populacional de Alouatta belzebul (Linnaeus, 1766) reintroduzidos na Reserva Biológica Guaribas, Paraîba. In: A Primatologia no Brasil 13. Passos, F.C. \& Miranda, J.M.D. (eds.). SBPr; Curitiba, PR. P: 79- 88.

Gregorin R. Taxonomia e Variação Geográfica das espécies do Gênero Alouatta Lacépéde (Primates, Atelidae) no Brasil. Revista Brasileira de Zoologia, 23(1): 64-144, 2006.

Hue T, Caubet M, Moura ACA. Howlers and marmosets in Pacatuba: an overcrowded existence in a semideciduous Atlantic forest fragment? Mammalia, 1-10, 2016.

IBAMA (Instituto Brasileiro de Meio Ambiente e dos Recursos Naturais Renováveis). 2003. Plano de Manejo Reserva Biológica Guaribas. MMA/IBAMA. 520p

ICMBIO (Instituto Chico Mendes de Conservação da Biodiversidade). 2020. <https://www.icmbio.gov.br/ portal/faunabrasileira/plano-de-acao-nacional-lista/865pan-primatas-do-nordeste > . Acesso em: 11/11/2020.

IMBIO (Instituto Chico Mendes de COnservação da Biodiversidade). 2021. < https://www.icmbio.gov.br/portal/ monitoramento-2016/programas-de-monitoramento-dabiodiversidade-em-ucs > . Acesso em: 18/03/2021.

Julliot C. Seed dispersal by red howling monkeys Alouatta seniculus on the seedling population in the understorey of tropical rain forest. International Journal of Primatology, 17: 239- 258, 1996.
Lopes MA, Ferrari, SF. Effects of human colonization on the Abundance and Diversity of Mammals in Eastern Brazilian Amazonia. Society for Conservation Biology, Vol. 14, 1658-1655, 2000.

MMA (Ministério do Meio Ambiente) 2014. Portaria 444 de 17 de dezembro de 2014. Diário Oficial da União 245 - Seção 1: 121- 126.

Neville MK, Glander KE, Braza F, Rylands AB. The howling monkeys, genus Alouatta. 1988. In: Mittermeier, R.; Rylands, A.B.; Coimbra Filho, A. \&amp; Fonseca,G.A.B. (eds.) Ecology and Behavior of Neotropical Primates. Vol. 2, WWF, Washington D.C., p.: 349-453.

Oliveira VB, Linares AM, Corrêa GLC, Chiarello AG. Predation on the black capuchin monkey Cebus nigritus (Primates, Cebidae) by domestic dogs Canis lupus familiaris (Carnivora, Canidae), in the Parque Estadual do Brigadeiro, Minas Gerais, Brazil. Revista Brasileira de Zoologia, 25 (2): 376- 378, 2008.

Peres C, Cunha AA. 2011. Manual para censo e Monitoramento de vertebrados de médio e grande porte por transecção linear em florestas tropicais. Wildlife Conservation Society, Ministério do Meio Ambiente. 44p.

Santos Jr., AP \& Buss, G. 2016. Abundância populacional do guariba-de-mãos-ruivas (Alouatta belzebul) na Área São João-Cafundó, Paraîba. Anais do VIII Seminário de Pesquisa e VIII Encontro de Iniciação Científica do ICMBio. Brasilia, DF, p. 26.

Souza, SP. 2005. Ecologia e conservação de Alouatta belzebul belzebul (Primates, Atelidae) na Paraiba, Brasil. Tese de Doutorado, UFMG, Belo Horizonte, MG. 121p.

Valença-Montenegro M, et al. 2018. Alouatta belzebul (Linnaeus, 1766). In: Livro Vermelho da Fauna Brasileira Ameaçada de Extinção. Volume 2 - Mamíferos. ICMBio/ MMA, Brasilia, DF, p. 148-152.

Zanella FCV. Evolução da biota da diagonal de formações abertas secas da América do Sul. Biogeografia da América do Sul. Padrões e Proc. Roca, p. 198- 220, 2011.

\section{Biodiversidade Brasileira - BioBrasil. Edição Temática: PIBIC n. 1,2022}

http://www.icmbio.gov.br/revistaeletronica/index.php/BioBR

Biodiversidade Brasileira é uma publicação eletrônica científica do Instituto Chico Mendes de

Conservação da Biodiversidade (ICMBio) que tem como objetivo fomentar a discussão e a disseminação de experiências em conservação e manejo, com foco em unidades de conservação $e$ espécies ameaçadas.

ISSN: 2236-2886 